ORIGINAL ARTICLE

\title{
Thyroid transcription factor 1 in pulmonary adenocarcinoma
}

\author{
G Stenhouse, N Fyfe, G King, A Chapman, K M Kerr
}

J Clin Pathol 2004;57:383-387. doi: 10.1136/jcp.2003.007138

See end of article for authors' affiliations .....................

Correspondence to: Dr K M Kerr, Department of Pathology, University Medical Building, University of Aberdeen, Aberdeen Royal Infirmary, Foresterhill, Aberdeen AB25 2ZD, UK; k.kerr@abdn.ac.uk

Accepted for publication 27 November 2003

\begin{abstract}
Aims: To discover whether variations in thyroid transcription factor 1 (TTF-1) staining in different subtypes and patterns of pulmonary adenocarcinoma are related to the putative origin of the tumour. In addition, to confirm the specificity of TTF-1 for pulmonary (as opposed to other sites) adenocarcinoma, to examine the possible prognostic relevance of TTF-1 positivity in lung cancer, and to review this laboratory's experience of TTF-1 in diagnostic practice.

Materials/Methods: In total, 128 primary lung adenocarcinomas, 106 primary non-pulmonary adenocarcinomas, and 37 pulmonary non-adenocarcinoma tumours were studied. In addition, 100 cases where TTF-1 was used in routine surgical pathology practice were investigated. Immunoperoxidase staining was performed on formalin fixed, paraffin wax embedded sections using anti-TTF-1 antibody. Staining was evaluated semiquantitatively using the frequency and intensity of nuclear positivity.

Results: None of the 106 non-pulmonary adenocarcinomas expressed TTF- 1 and only three of the 37 nonadenocarcinoma lung cancers, all neuroendocrine carcinomas, were positive. Of the pulmonary adenocarcinomas, $75 \%$ were strongly positive for TTF-1. Mucinous (two of six) and poorly differentiated adenocarcinomas (four of 10) were less likely to stain. Of the peripheral adenocarcinomas, 33 of 37 were positive, whereas only seven of 14 of those of bronchial origin stained strongly. Atypical adenomatous hyperplasia strongly expressed TTF-1. No "false positives" were encountered in the 100 routine diagnostic cases.

Conclusion: Positive TTF-1 staining is useful in the differential diagnosis of pulmonary adenocarcinomas. TTF-1 may be a lineage marker for tumours arising from the peripheral airway or alveolar epithelium and has no prognostic relevance.
\end{abstract}

ung cancer is a common, frequently fatal, malignancy and the adenocarcinoma subtype is rising in -incidence in many countries. It is more prevalent in women and, overall, is now the most common form of lung cancer in many parts of the world. ${ }^{12}$ Adenocarcinomas of the lung readily metastasise and, as the diversity of chemotherapeutic agents increases, with differential efficacy against cancers from different sites, the importance of the histopathologist being able to ascertain the primary source of a metastatic tumour has increased.

Thyroid transcription factor 1 (TTF-1) is a tissue specific, homeodomain containing transcription factor, which is found predominantly in normal type II alveolar pneumocytes. It is also present in thyroid tissue and has been identified during the embryological development of human lung and brain. ${ }^{3-5}$ TTF-1 plays a role in epithelial morphogenesis, ${ }^{6}$ stimulates the synthesis of pneumocyte surfactant proteins, and regulates secretory product gene transcription in Clara cells. ${ }^{67}$

Among non-small cell carcinomas of lung, TTF-1 has been found in up to $76 \%$ of adenocarcinomas but rarely, if ever, in squamous carcinomas. ${ }^{8-11}$ In several tissues and sites where metastatic carcinoma has been found in biopsy material, anti-TTF-1 immunohistochemistry has been useful in the differentiation of metastatic, morphologically similar, adenocarcinomas of pulmonary origin from other unknown primary sites. ${ }^{12-16}$ The lung is also a common site for metastases, and solitary metastatic deposits may be resected, as targeted treatment for limited metastatic disease, or in the belief that the tumour is primary in the lung.
"As the diversity of chemotherapeutic agents increases, with differential efficacy against cancers from different sites, the importance of the histopathologist being able to ascertain the primary source of a metastatic tumour has increased"

In addition to the expected expression in thyroid tumours, TTF-1 has also been demonstrated in small cell carcinomas and other neuroendocrine tumours. ${ }^{17-21}$ Although most studies have suggested that TTF-l can be found in neuroendocrine tumours of both pulmonary and extrapulmonary origin, ${ }^{11} 1819$ some have found the marker largely confined to those arising in the lung..$^{21}$

In our study, we examined TTF-1 reactivity in 165 pulmonary carcinomas and 106 non-pulmonary adenocarcinomas. Specifically, we considered the different architectural subtypes (as described in the recent World Health Organisation (WHO)/International Association for the Study of Lung Cancer (IASLC) classification, ${ }^{22}$ ) exhibited by the adenocarcinomas, to ascertain whether or not TTF-1 expression varies between them. Given the propensity for TTF-1 staining in alveolar pneumocytes and Clara cells, when compared with the bronchial epithelium, ${ }^{5}$ we also hypothesised that peripheral-type adenocarcinomas would show more positivity than central bronchial-type adenocarcinomas. ${ }^{23}{ }^{24}$ Furthermore, we reviewed the usefulness of antiTTF-1 staining in 100 consecutive diagnostic surgical histopathology/cytopathology cases, where its use was aimed

Abbreviations: AAH, atypical adenomatous hyperplasia; BAC, bronchioloalveolar carcinoma; TTF-1, thyroid transcription factor 1; WHO, World Health Organisation 
at the identification of pulmonary adenocarcinoma in a range of clinicopathological situations.

\section{MATERIALS AND METHODS Study material}

TTF- 1 reactivity was studied in 271 primary tumours. Of 165 resected primary pulmonary tumours, 128 were adenocarcinomas, 29 were squamous, four were mixed tumours, one was large cell undifferentiated, two were large cell neuroendocrine carcinomas, and one was a typical carcinoid tumour. A further 106 primary non-pulmonary adenocarcinomas of the pancreas (seven), endometrium (25), ovary (15), stomach (24), and colon (35) were also studied. In each case, the archival surgical histopathological material was examined and an appropriate tissue block selected for sectioning and staining. In the case of the pulmonary adenocarcinomas, in the section selected for immunostaining, the range of different tumour patterns present, as defined in the 1999 WHO/IASLC classification, ${ }^{22}$ namely solid, tubular, papillary, mucinous, and bronchioloalveolar (BAC), was recorded and, using information available in the original report and by study of all the available sections, a judgement was made as to whether the tumour was of peripheral parenchymal type, central bronchial type, or unclassifiable. ${ }^{23}{ }^{24}$ Central bronchial-type adenocarcinomas frequently arise in conjunction with a large airway, are large, often well circumscribed tumours with focal necrosis, and may have an endobronchial component. Histologically, they consist of sheets or lobules of large cuboidal or columnar malignant cells, often with an acinar or cribriform pattern. Better differentiated tumours show round acini, and columnar cells with basal nuclei and eosinophilic cytoplasm. Mucin is variable, may be abundant, and some tumours have mucin containing signet ring cells. Parenchymal-type adenocarcinoma is usually a peripheral, subpleural lesion of variable size, often with pronounced pleural puckering and a central anthracotic scar. A peripheral BAC component is very common, whereas the invasive tumour is usually a heterogeneous mixture of irregular acini in a fibrous stroma, papillary tumour or more solid, poorly differentiated adenocarcinoma. The cell type is also a more heterogeneous mixture, including mucin secreting cells, goblet cells, columnar cells, and various rounded, ovoid, tongue shaped, and "peg" cells corresponding to Clara cell and type II pneumocyte differentiation. Occasionally, a focus of tumour with a central-type morphology may be seen.

A further 100 cases encompassed numerous tissues from biopsy material, postmortem tissues, and cytological preparations encountered in local routine diagnostic practice. AntiTTF-1 was used, in conjunction with a range of other antibodies, appropriate to the particular clinical problem, where lung adenocarcinoma entered the differential diagnosis. Note was taken of all clinical information available, up until the time of our study.

\section{Immunohistochemical staining}

Immunohistochemical staining was carried out on $4 \mu \mathrm{m}$ thick sections cut from formalin fixed, paraffin wax embedded tissue, using microwave antigen retrieval and a standard streptavidin-biotin based technique. ${ }^{25}$ The anti-TTF1 antibody (8G7G3/1, mouse monoclonal antibody; Dako, Ely, Cambridgeshire, UK) was used at a dilution of 1/100, with a positive and negative control for each assay. Immunoreactivity was evaluated semiquantitatively according to the percentage of cells displaying nuclear staining $(0$; ,$+ \leqslant 10 \%$ cells positive;,$++ 10-50 \%$ positive;,$+++>50 \%$ positive) along with the intensity of that staining (graded + to +++ ). Scoring was carried out independently by two of us (GS and KMK). These scores were then reviewed, a consensus was agreed upon in any case where there was disagreement, and a decision taken to allocate an overall score of low/negative or high staining. Cytoplasmic staining was not scored as positive. In each case of primary lung cancer examined, particular attention was paid to ensure that entrapped normal alveolar walls within the tumour were not erroneously scored as positive tumour.

Length of postoperative survival was available on all patients with primary lung carcinoma from our own lung cancer data base and the Scottish Cancer Registry.

\section{Statistics}

The $\chi^{2}$ test was used to examine the association between TTF1 staining in the bronchial and peripheral-type adenocarcinomas. The Kaplan-Meier method was used to test for differences in postoperative survival using log rank to indicate significance.

\section{RESULTS}

None of the 106 adenocarcinomas of non-pulmonary origin showed nuclear staining. Weak cytoplasmic positivity was seen in five cases (three gastric and two endometrial adenocarcinomas). Similarly, of the 37 pulmonary carcinomas, which were not adenocarcinoma, only the three neuroendocrine tumours (two large cell and one typical carcinoid tumour) showed evidence of strong nuclear staining.

Of the 128 primary pulmonary adenocarcinomas, 96 (75\%) had high level nuclear staining for TTF-1. Of the remaining 32 tumours $(25 \%), 14$ showed weak positivity whereas 18 were completely negative, giving an overall positivity rate of $86 \%$. Table 1 shows the expression of TTF- 1 by overall tumour type and table 2 displays staining by tumour location. Peripheral, parenchymal tumours and those with prominent BAC or papillary patterns stained most strongly. Central, bronchial tumours and those with the poorest differentiation stained the least. Analysis of the patterns of primary lung adenocarcinoma present in the 128 cases showed only one scorable pattern in 76 sections, a further 44 revealed two patterns, and eight had three. Table 3 shows the results of TTF-1 staining of the various patterns of adenocarcinoma. The mucinous carcinomas appear to stain less well for TTF-1, and three mucinous BACs were also negative. High positivity was noted in only four of the 10 poorly differentiated areas of tumour and was relatively low in "solid" adenocarcinoma, whereas the better differentiated patterns showed more staining (fig 1A, B). Areas with a non-mucinous bronchioloalveolar pattern and papillary tumour showed the strongest and most abundant staining for TTF-1 (fig lC).

Those bronchial-type adenocarcinomas $(n=14)$ were positive in half the cases, with generally only moderate staining, whereas 33 of the 37 peripheral tumours were positive $(p<0.02)$. In this last case, there was frequently widespread intense staining, often reflecting the presence, almost by definition, of BAC-like areas of tumour. Fortuitously, several sections of pulmonary adenocarcinoma also had foci of atypical adenomatous hyperplasia (AAH) in adjacent lung. Each of these five separate AAH lesions was strongly positive for TTF-1 (fig 1D).

Survival analysis for those patients with primary lung cancer showed no relation between TTF-1 expression and length of postoperative survival.

Specimens that had been stained for TTF-1 in routine surgical histo/cytopathology practice comprised 76 surgical histopathology cases from lung, bronchus, pleura, intestine, bone marrow, lymph node, brain, peritoneum and omentum, together with 20 cytopathology specimens of pleural or peritoneal fluid, or fine needle aspiration biopsy of several sites. Four postmortem cases were also investigated. To the 

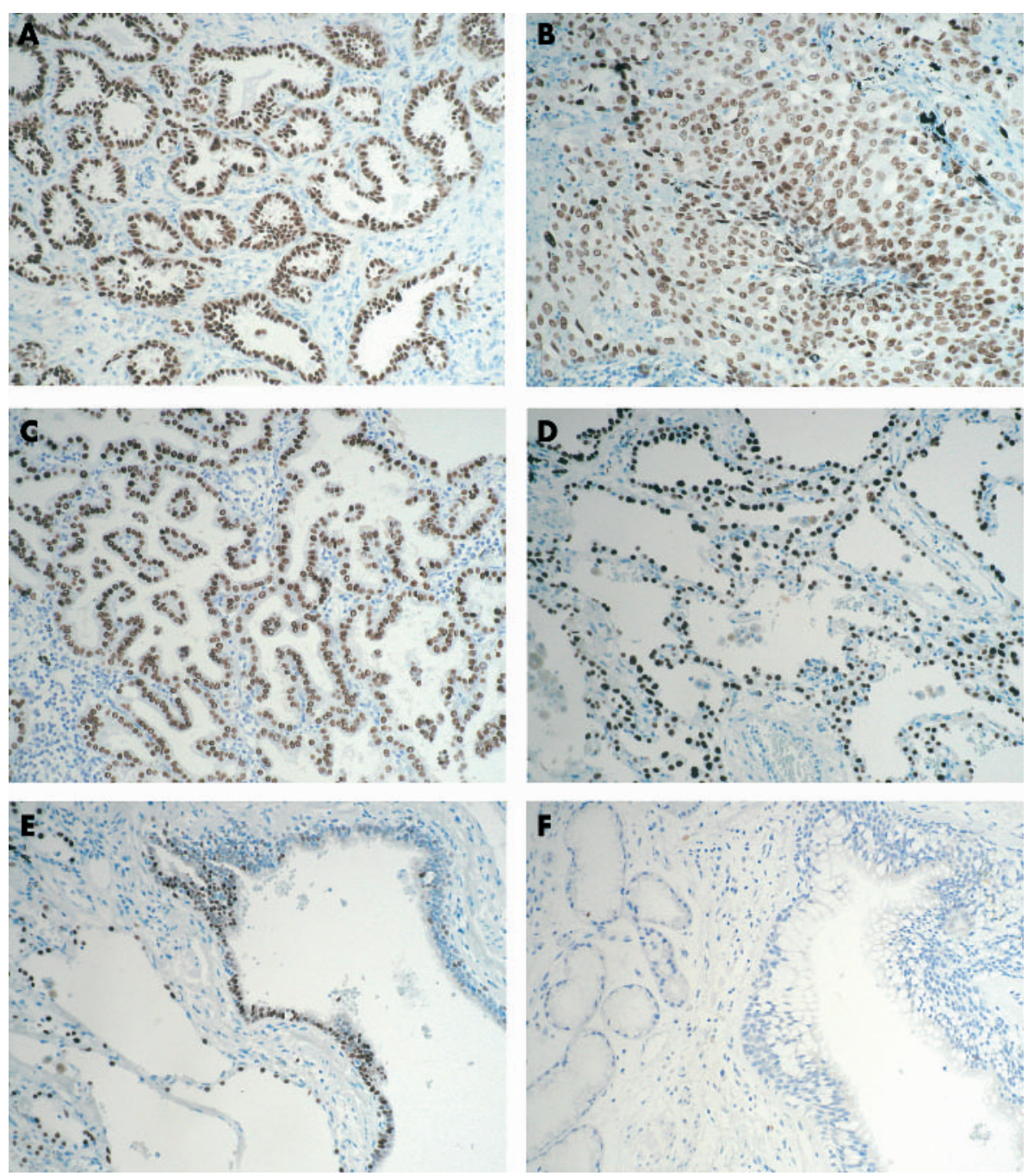

Figure 1 (A) Invasive acinar pattern of adenocarcinoma showing strong staining for thyroid transcription factor 1 (TTF-1). (B) Solid, more poorly differentiated adenocarcinoma exhibiting a high degree of staining. (C) Abundant staining in non-mucinous bronchioloalveolar carcinoma. (D) Strong positivity in atypical adenomatous hyperplasia. (E) Alveolar pneumocytes and bronchiolar epithelium are positive for TTF-1. In bronchioles staining is much stronger where the epithelium shows less stratification. (F) Central bronchial epithelium negative for TTF-1. best of our knowledge, using all the information available to us, no inappropriate "false positives" occurred in the adenocarcinomas. There were 15 positive cases where the diagnosis was pulmonary adenocarcinoma. Nine positive tumours, which were patently not primary pulmonary adenocarcinoma, were shown to be of thyroid origin or neuroendocrine carcinomas by other methods. Staining was absent in tumours from two primary lung adenocarcinomas, one of which was poorly differentiated, the other probably of bronchial origin. Seventy four cases were judged to be "true

Table 1 TTF-1 staining in different tumour subtypes

\begin{tabular}{ll}
\hline Overall adenocarcinoma subtype (WHO) & High TTF-1 staining \\
\hline Mixed pattern & $55 / 65(85 \%)$ \\
Non-mucinous BAC & $9 / 9(100 \%)$ \\
Acinar, papillary, or tubular & $10 / 14(71 \%)$ \\
Solid with mucin & $14 / 25(56 \%)$ \\
Variants & $3 / 6(50 \%)$ \\
Mucinous (including mucinous BAC) & $1 / 1(100 \%)$ \\
Signet ring cell & $1 / 2(50 \%)$ \\
Clear cell & $3 / 6(50 \%)$ \\
Pleomorphic/sarcomatoid with & \\
adenocarcinoma & \\
\hline In the mixed category, a minimum of 10\% of each component was \\
present in the tumour ( $\mathrm{n}=128)$. \\
BAC, bronchioloalveolar carcinoma; TTF-1, thyroid transcription factor $1 ;$ \\
WHO, World Health Organisation.
\end{tabular}

negative" cases; that is, TTF-1 negative and the absence of lung adenocarcinoma.

\section{DISCUSSION}

Overall, $75 \%$ of primary pulmonary adenocarcinomas showed strong staining for TTF-1, and a further $11 \%$ showed weak staining. Apart from a few neuroendocrine tumours, none of the other primary lung cancers and none of 106 nonpulmonary adenocarcinomas showed staining for TTF-1. In lung adenocarcinomas, the papillary and BAC patterns were more likely to stain for TTF-1, whereas poorly differentiated and solid adenocarcinomas were least likely.

Our score for substantial TTF-1 staining in $75 \%$ of lung adenocarcinomas agrees well with the findings of several previous reports, where the range of positivity was 72$80 \%,{ }^{9} 102627$ although there are exceptions, with one study

Table 2 Thyroid transcription factor 1 (TTF-1) staining by tumour origin, either central, peripheral parenchymal, or indeterminate $(n=128)$

\begin{tabular}{ll}
\hline Parenchymal $\boldsymbol{v}$ bronchial & High TTF-1 staining \\
\hline Peripheral, parenchymal type & $33 / 37(89 \%)$ \\
Indeterminate group & $56 / 77(73 \%)$ \\
Central, bronchial type & $7 / 14(50 \%)$ \\
\hline
\end{tabular}


Table 3 Number of areas, identified in 128 different adenocarcinomas, showing a specific architectural subtype, with number showing high positive staining for TTF-1

\begin{tabular}{lll}
\hline Subtype pattern & No. of areas & High positive \\
\hline NMBAC & 33 & $32(97 \%)$ \\
MBAC & 4 & $1(25 \%)$ \\
Papillary & 11 & $11(100 \%)$ \\
Pap/Tub & 18 & $15(83 \%)$ \\
Tubular & 48 & $40(83 \%)$ \\
Solid & 58 & $42(72 \%)$ \\
PD & 10 & $4(40 \%)$ \\
Mucinous & 6 & $2(33 \%)$ \\
Total & 188 & $147(78 \%)$ \\
\hline
\end{tabular}

MBAC, mucinous bronchioloalveolar carcinoma; NMBAC, non-mucinous bronchioloalveolar carcinoma; Pap/Tub, admixed papillary and tubular patterns, where the papillary and tubular components could not be separated for the purpose of scoring; PD, poorly differentiated (including pleomorphic and sarcomatous areas).

reporting only $25 \%$ positivity. ${ }^{11}$ It is likely that this unusual finding results from technical differences in the anti-TTF-1 antibody and staining methodologies used. Our data also reinforce the almost universal finding that anti-TTF-1 only stains adenocarcinomas that are primary in the lung and thyroid gland. ${ }^{10} 2728$

The issue of the staining pattern and its relation to tumour type and differentiation has been addressed to a variable extent in some previously published reports. Several studies have reported that there was no correlation between TTF-1 staining and tumour growth pattern or differentiation. ${ }^{91126}$ Our data suggest that poorly differentiated tumours are less likely to be strongly positive than relatively well differentiated lesions showing complex architecture such as glands, tubules, papillae, or a BAC-like growth pattern. Care was taken throughout our study to ensure that issues such as poor fixation in the centre of a large tumour did not account for a false negative result. There are reports showing that mucinous tumours of the lung do not express TTF-1. Some have found, as we did, that mucinous BAC is usually negative for TTF- $1,{ }^{28}{ }^{29}$ whereas invasive mucinous tumours show little positivity. ${ }^{10}$ Interestingly, the rare signet ring cell mucin secreting carcinoma seems to express abundant amounts of TTF- $1 .{ }^{30}{ }^{31}$ Our finding that $50 \%$ of pleomorphic, sarcomatoid tumours are also positive is in keeping with a previous study. ${ }^{32}$

\section{"Our data suggest that poorly differentiated tumours are less likely to be strongly positive than relatively well differentiated lesions showing complex architecture"}

Most adenocarcinomas are believed to arise in the peripheral parts of the lung, from alveolar or bronchioloalveolar epithelium, possibly through a preneoplastic precursor lesion, such as $\mathrm{AAH}^{33}{ }^{34} \mathrm{~A}$ smaller number are thought to arise centrally from bronchial epithelium. ${ }^{23} 2435$ Our attempts to classify these tumours according to the bronchial versus peripheral division resulted in a large proportion of unclassifiable cases $(60 \%)$, much higher than Edwards' figure of $20 \%$. Our proportion of bronchial-type cases was similar to Edwards. It is probable that many of our indeterminate group are peripheral-type tumours (this may be inferred in part by the fact that $73 \%$ of these showed abundant TTF-1 staining), which were excluded by strict interpretation of the criteria. Although TTF-1 expression is universal and strong in alveolar epithelium and generally strong and frequent in more peripheral bronchiolar epithelium, it is generally absent in larger bronchioles and in the central

\section{Take home messages}

- Staining for thyroid transcription factor 1 (TTF-1) is useful in the differential diagnosis of pulmonary adenocarcinomas if other tumours known to express this protein (thyroid and neuroendocrine tumours) are excluded

- TTF-1 may be a lineage marker for tumours arising from the peripheral airway or alveolar epithelium and reflects the degree of tumour differentiation

- TTF-1 had no prognostic relevance in our series

bronchial epithelium (fig 1E, F). ${ }^{5}{ }^{10}$ This reflects the association between TTF- 1 and both alveolar pneumocyte and Clara cell secretory activity. ${ }^{67}$ The finding of greater staining in non-mucinous BAC-type areas of tumour and peripheral parenchymal-type adenocarcinomas, when compared with those deemed to be of central bronchial type, seems to reflect the types of cytodifferentiation prevalent in different tumour types and, perhaps, the probable origin of the tumour. This supports the assertion of Yatabe et al, who suggested that TTF- 1 expression could be used as a lineage marker for the terminal respiratory unit. ${ }^{36}$ Thus, it is not surprising that the five examples of AAH stained by us showed strong expression of TTF-1.

We were unable to show that absence of TTF-1 staining was related to worse survival in a substantial group of surgically resected patients. Such an association has been demonstrated by Haque et al. ${ }^{26}$ It is not clear whether this is a true relation or a finding compounded by tumour size and grade.

Our review of the usefulness of TTF-1 as a marker of pulmonary adenocarcinoma in surgical histo/cytopathological diagnosis has shown that it is indeed extremely useful, with no known case of a false positive. This is reflected in the literature. $^{8}{ }^{13-15}$ A few false negative cases occurred in our review, but this is not unexpected given the possibility of sampling problems and our finding that poorly differentiated, solid pattern and central bronchial-type adenocarcinomas stain less well for TTF-1.

TTF-1 is a useful and reliable marker for pulmonary adenocarcinoma, in circumstances where other tumours known to express this protein (thyroid and neuroendocrine tumours) are excluded. TTF-1 expression does, to some extent, reflect the pattern(s) and degree of differentiation evident in the tumour and may well be dependent on the site of origin and histogenesis of the tumour.

\section{ACKNOWLEDGEMENTS}

This work was supported by grants from the Grampian University Hospitals NHS Trust Endowment Funds, the Aberdeen Lung Cancer Group, and the "Friends of Anchor" Fund. We thank Dr M Kennedy and Mr R Jeffrey for their work in helping establish our Grampian Pulmonary Resection Database.

\section{Authors' affiliations}

G Stenhouse, N Fyfe, G King, A Chapman, K M Kerr, Aberdeen Royal Infirmary, Foresterhill, Aberdeen AB25 2ZD, UK

\section{REFERENCES}

1 Travis WD, Travis LB, Devesa SS. Lung cancer. Cancer 1995:75:191-201.

2 Harkness EF, Brewster DH, Kerr KM, et al. Changing trends in incidence of lung cancer by histologic type in Scotland. Int J Cancer 2002;102:179-83.

3 Ikeda K, Clark JC, Shaw-White JR, et al. Gene structure and expression of human thyroid transcription factor-1 in respiratory epithelial cells. J Biol Chem 1995;270:8108-14. 
4 Lazzaro $D$, Price $M$, de Felice $M$, et al. The transcription factor TF-1 is expressed at the onset of thyroid and lung morphogenesis and in restricted regions of the foetal brain. Development 1991;113:1093-104.

5 Stahlman MT, Gray ME, Whitsett JA. Expression of thyroid transcription factor-1 (TTF-1) in fetal and neonatal lung. J Histochem Cytochem 1996:44:673-8.

6 Minoo P, Hamdan $H, B u D$, et al. TTF-1 regulates lung epithelial morphogenesis. Dev Biol 1995; 172:694-8.

7 Zhang L, Whitsett JA, Stripp BR. Regulation of Clara cell secretory protein gene transcription by thyroid transcription factor-1. Biochim Biophys Acta 1997; 1350:359-67.

8 Lau SK, Luthringer DJ, Eisen RN. Thyroid transcription factor-1: a review. Appl Immunohistochem Mol Morphol 2002;10:97-102.

9 Pelosi G, Fraggetta F, Pasini F, et al. Immunoreactivity for thyroid transcription factor-1 in stage I non-small cell carcinomas of the lung. Am J Surg Pathol $2001 ; 25: 363-72$

10 Kaufmann O, Dietel M. Thyroid transcription factor- 1 is the superior immunohistochemical marker for pulmonary adenocarcinomas and large cell carcinomas compared to surfactant proteins A and B. Histopathology 2000;36:8-16

11 Fabbro D, Di Loreto C, Stamerra O, et al. TFF-1 gene expression in human lung tumours. Eur J Cancer 1996;32A:512-17.

12 Turbat-Herrera EA, Zhu W. Diagnostic cytopathology: refining diagnoses with the use of ancillary techniques. Pathology Case Reviews 2002; 7:173-85.

13 Ordoñez NG. Value of thyroid transcription factor-1, E-cadherin, BG8, WTI, and CD44S immunostaining in distinguishing epithelial pleural mesothelioma from pulmonary and nonpulmonary adenocarcinoma. Am J Surg Pathol 2000;24:598-606.

14 Bohinski RJ, Bejarano PA, Balko G, et al. Determination of lung as the primary site of cerebral metastatic adenocarcinomas using monoclonal antibody to thyroid transcription factor-1. J Neurooncol 1998;40:227-31.

15 Sheppard MN. Specific markers for pulmonary tumours. Histopathology 2000;36:273-6.

16 Silverman JF. Effusion cytology of metastatic malignancy of unknown primary. Pathology Case Reviews 2001;6:154-60.

17 Di Loreto C, Di Lauro V, Puglisi F, et al. Immunocytochemical expression of tissue specific transcription factor-1 in lung carcinoma. J Clin Pathol 1998;50:30-2

18 Cheuk W, Kwan MY, Suster S, et al. Immunostaining for thyroid transcription factor-1 and cytokeratin 20 aids the distinction of small cell carcinoma from Merkel cell carcinoma, but not pulmonary from extrapulmonary small cell carcinomas. Arch Pathol Lab Med 2001;125:228-31.

19 Agoff SN, Lamps LW, Philip AT, et al. Thyroid transcription factor-1 is expressed in extrapulmonary small cell carcinomas but not in other extrapulmonary neuroendocrine tumours. Mod Pathol 2000;13:238-42.
20 Oliveira AM, Tazelaar HG, Myers JL, et al. Thyroid transcription factor-1 distinguishes metastatic pulmonary from well-differentiated neuroendocrine tumours of other sites. Am J Surg Pathol 2001;25:815-19.

21 Ordoñez NG. Value of thyroid transcription factor-1 immunostaining in distinguishing small cell lung carcinoma from other small cell carcinomas. Am J Surg Pathol 2000;24:1217-23.

22 Travis WD, Colby TV, Corrin B, et al. Histological typing of lung and pleural tumours. WHO international histological classification of tumours, 3rd ed. Berlin: Springer, 1999.

23 Edwards CW. Pulmonary adenocarcinoma: review of 106 cases and proposed new classification. J Clin Pathol 1987;40:125-35.

24 Shimosato Y. Bronchial gland type adenocarcinoma. In: Corrin B, ed. Pathology of lung tumours. Edinburgh: Churchill Livingstone, 1997:135-48.

25 King G. Microwave heating as a method of unmasking immunoglobulin light chain antigen in paraffin sections. Immunocytochemistry News chain antigen in
$1994 ; 3: 11-12$.

26 Haque AK, Syed S, Lele SM, et al. Immunohistochemical study of thyroid transcription factor-1 and HER2/neu expression in non-small cell lung cancer: strong thyroid transcription factor-1 expression predicts better survival. Appl Immunohistochem Mol Morphol 2002; 10:103-9.

27 Bejarano PA, Baughman RP, Biddinger PW, et al. Surfactant proteins and thyroid transcription factor-1 in pulmonary and breast carcinomas. Mod Pathol 1996;9:445-52.

28 Goldstein NS, Thomas M. Mucinous and nonmucinous bronchioloalveolar adenocarcinoma has distinct staining patterns with thyroid transcription factor and cytokeratin 20 antibodies. Am J Clin Pathol 2001;1 16:319-25.

29 Lau SK, Desrochers MJ, Luthringer DJ. Expression of thyroid transcription factor- 1 , cytokeratin 7 , and cytokeratin 20 in bronchioloalveolar carcinomas: an immunohistochemical evaluation of 67 cases. Mod Pathol 2002; 15:538-42.

30 Castro CY, Moran DG, Flieder DG, et al. Primary signet ring cell adenocarcinomas of the lung: a clinicopathological study of 15 cases. Histopathology 2001;39:397-401.

31 Merchant SH, Amin MB, Tamboli P, et al. Primary signet-ring cell carcinoma of lung: immunohistochemical study and comparison with non-pulmonary signet-ring cell carcinomas. Am J Surg Pathol 2001;25:1515-9.

32 Rossi G, Cavazza A, Sturm N, et al. Pulmonary carcinomas with pleomorphic, sarcomatoid, or sarcomatous elements: a clinicopathologic and

immunohistochemical study of 75 cases. Am J Surg Pathol 2003;27:31 1-24.

33 Kerr KM. Adenomatous hyperplasia and the origin of peripheral adenocarcinoma of the lung. In: Corrin B, ed.Pathology of lung tumours. Edinburgh: Churchill Livingstone, 1997:119-34.

34 Kerr KM. Pulmonary preinvasive neoplasia. J Clin Pathol 2001;54:257-71.

35 Cooper CA, Carey FA, Bubb VJ, et al. The pattern of K-ras mutation in pulmonary adenocarcinoma defines a new pathway of tumour development in the human lung. J Pathol 1997;181:401-4.

36 Yatabe Y, Mitsudomi T, Takahashi T. TTF-1 expression in pulmonary adenocarcinomas. Am J Surg Pathol 2002;26:767-73. 